\title{
X-ray measurements of plunging breaking solitary waves
}

\author{
Lisa Smith ${ }^{\mathrm{a}, *}$, Jostein Kolaas ${ }^{\mathrm{a}}$, Atle Jensen ${ }^{\mathrm{a}}$, Kristian Sveen ${ }^{\mathrm{a}, \mathrm{b}}$ \\ ${ }^{a}$ Department of Mathematics, University of Oslo, Norway \\ ${ }^{b}$ Institute for Energy Technology, Norway
}

\begin{abstract}
The aim of this study is to examine and measure the characteristics of air cavities generated by breaking solitary waves by utilizing a novel tomographic X-ray system. Small scale experiments of solitary waves that propagate on a $5.1^{\circ}$ (1:10) beach are conducted. Waves with amplitude normalized with the water depth, $a / H=0.47$ on a flat bottom are investigated by two perpendicular X-ray systems. Images are captured at locations from the surf zone to the swash zone and at maximum runup. A large air tube is observed right after the plunger impacts the dry beach. Void velocity and the shape of the large air tube, are measured and reported. The large air tube evolves from a symmetrical shape with two large air pockets located close to the walls of the wave tank, to an asymmetrical shape. Contrast enhanced X-ray images reveal that the swash tongue surface is unstable and that secondary mixing of air and water occurs. X-ray images from the maximum runup reveal that the air is still entrapped by the thin swash tongue at times close to maximum runup.
\end{abstract}

\section{Introduction}

At sea, wave breaking contributes to transport and mixing of air and seawater. The amount of air and water transferred through the interface are important mechanisms for heat exchange, vapor generation and gas dissolution in the ocean. The amount of $\mathrm{CO}_{2}$ dissolved in the ocean and the amount of vapor in the atmosphere are known to affect the weather and the climate (Banner and Peregrine, 1993). On small scale, the air entrained and dissolved by breaking may affect the quality and acidity of the water, which is crucial for marine life. Close to the shore, wave breaking is related to the effect of air entrainment on dissipation, momentum and mass fluxes, where the latter is important for erosion and sediment transport on shore. It may also be responsible for large deformation and destruction of installations located in the near shore region. Although the abundance of application of wave breaking, many features are still poorly understood.

Large waves that travel on a sloping beach will steepen, and may reach a critical level where the waves break. The breaking may result in overturning jets, white caps, sprays, air bubbles entrainment and rough surfaces. Four different types of wave breaking are defined: spilling, plunging, surging and collapsing breakers (Peregrine, 1983). Grilli et al. (1997) characterized the different breaker types based on a surf similarity parameter $S_{o}=1.521 \frac{\tan \alpha}{\sqrt{H_{o}}}$ for solitary waves. The waves that generate an overturning jet are called plunging breakers and are reported to have a similarity parameter between 0.025 and 0.3 . Another description of the plunging breaking waves was given by Longuet-Higgins (1982). They fitted the overturning jet to a cubic spline function and found that the aspect ratio of the curve omitting the air underneath the plunge $l / w$ was 2.75 (see Figure 1). Both measurements (Lamarre and Melville (1991), Blenkinsopp and Chaplin (2007, 2008)), and computation (Iafrati (2009)) of the ratio of the cavity size to the breaker height $A / H_{p}^{2}$ is reported to range from 0.0291 to 0.4 . A is the area underneath the overturning jet and the $H_{p}$ is the wave height when the jet impinges the beach or reattaches with the water. When the plunging jet impinges the still water or the beach, it may splash and additional air may be entrained into the water. Cycles of smaller splashing events can arise from this large splash event (Peregrine, 1983). This may also increase the amount of air encapsulated by the water. After breaking, turbulent motion of the surface may also lead to air entrainment

*lisasmi@math.uio.no 
(Kiger and Duncan, 2012). These complex processes make it difficult to understand, and measure the amount of air entrained in a breaking swash event.

In the latest years several studies on runup of solitary waves have been conducted (Pedersen et al. (2013) and Smith et al. (2017)). The long single crested solitary waves are easy to work with experimentally since they are described by a single parameter A/H. Optical measurement techniques such as Particle Image Velocimetry (PIV), Particle Tracking Velocimtery (PTV), and Laser Doppler Velocimetry (LDV) are widely used to investigate velocities, acceleration and turbulent structures in breaking events (Jensen et al. (2003), Lin et al. (2015), Chang and Liu (1998) and Smith et al. (2017)). In the latter study, bubble information on the large air bubble encapsulated by a plunging breaker was retrieved from an optical image technique. Difficulties were reported with detection of the large air bubble for the strongest plunger $A / H \approx 0.50$, and were due to irregularities in the bubble shape. This resulted in light distortion and limited optical access that prevented measurement of the air cavity.

Micro scale wave breaking is investigated by Siddiqui et al. (2001). They compared infra-red light measurements and optical PIV measurements and found a correlation between the thermal processes and vortexes generated during breaking. At the other end of the spectra, X-rays are mostly utilized in medical contexts, but are recently used to investigate fluid flows ( Heindel et al. (2008)). Monochromatic X-ray attenuation is defined by Beers law (Wellington et al., 1987) :

$$
\frac{I}{I_{0}}=\exp \left(-\mu l_{x}\right)
$$

where $I_{0}$ is the incident X-ray intensity, $I$ is intensity after the X-rays have propagated through a material of length $l_{x}$ and with attenuation coefficient $\mu$. This principal was used by $\mathrm{Hu}$ et al. (2005) and $\mathrm{Hu}$ et al. (2014) to investigate surface structures and void fractions in multiphase pipe flow.

New developments in detector microelectronics and recent achievements in manufacturing of X-ray sources have made it possible to record X-ray images with both high temporal and spatial resolution. This enables us to investigate strongly time dependent phenomenon such as wave breaking in great detail. The use of $\mathrm{X}$-ray techniques instead of optical techniques is beneficial when opaque flows are investigated. The present of air bubbles in experiments affects the optical accessibility due to reflection and refraction of light rays at the bubbles interfaces. Smith et al. (2016) used tomographic X-ray technique to measure surface elevation of small scale solitary waves, and compared the measurements to ultrasonic gauges measurements. The study showed excellent agreement between the measuring techniques, and the X-ray visualization revealed a capillary cross wave located on top of the solitary wave crest, with an amplitude of approximately $2 \mathrm{~mm}$. The present study is a continuation of the experiment conducted in Smith et al. (2016), but improvements of the X-ray visualization technique are conducted, and more advance measurements are achieved. The wave generation mechanism is upgraded, and a beach is inserted at the end of the wave tank to induce wave breaking. The purpose of this study is to demonstrate a novel X-ray technique for detecting air cavities within a breaking event. In this paper, ultra fast X-ray measurements of solitary wave breaking are presented. The paper starts with a description of the experimental setup in Section 2, the X-ray settings in 2.1, the post-processing in 2.2 and a description of a simple 3D-reconstruction algorithm in 2.3. The results are provided in Section 3 where the measurements from the ultrasonic probes are given in Section 3.1, the $\mathrm{X}$-ray measurements are presented in Section 3.2 and the maximum runup results are given in 3.3. The 3Dreconstruction results are shown in Section 3.4. A discussion of the scale effects related to the experiments is provided in Section 4. Finally, a conclusion is presented in Section 5.

\section{Experimental setup and post processing}

Experiments are conducted at the Hydrodynamics lab at the University of Oslo. A $3 \mathrm{~m}$ long and $10 \mathrm{~cm}$ wide wave tank, filled with $5 \mathrm{~cm}$ water, is mounted in an X-ray cabinet. The wave tank is placed in the middle of the region spanned by two perpendicular X-ray systems. A sketch of the experiment is shown in Figure 2. A block releasing mechanism generates solitary waves with an amplitude normalized by the water depth of $a / H=0.47$. The block is made out of polymer, and has a piece of magnetic metal attached on top. A strong magnet holds the block slightly below the water level until an electrical signal switches it off. The polymer block is then released into the water and a solitary wave is generated. The dimensions of the block are $15.03 \mathrm{~cm}, 4.93 \mathrm{~cm}$ and $5.00 \mathrm{~cm}$ (length, width, height). A banner $18 \mathrm{U}$-sage ultrasonic wave gauge 


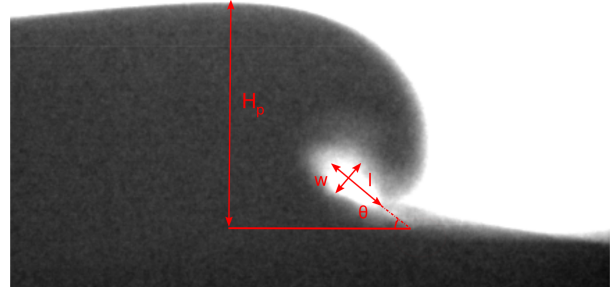

Figure 1: Vortex shape parameters

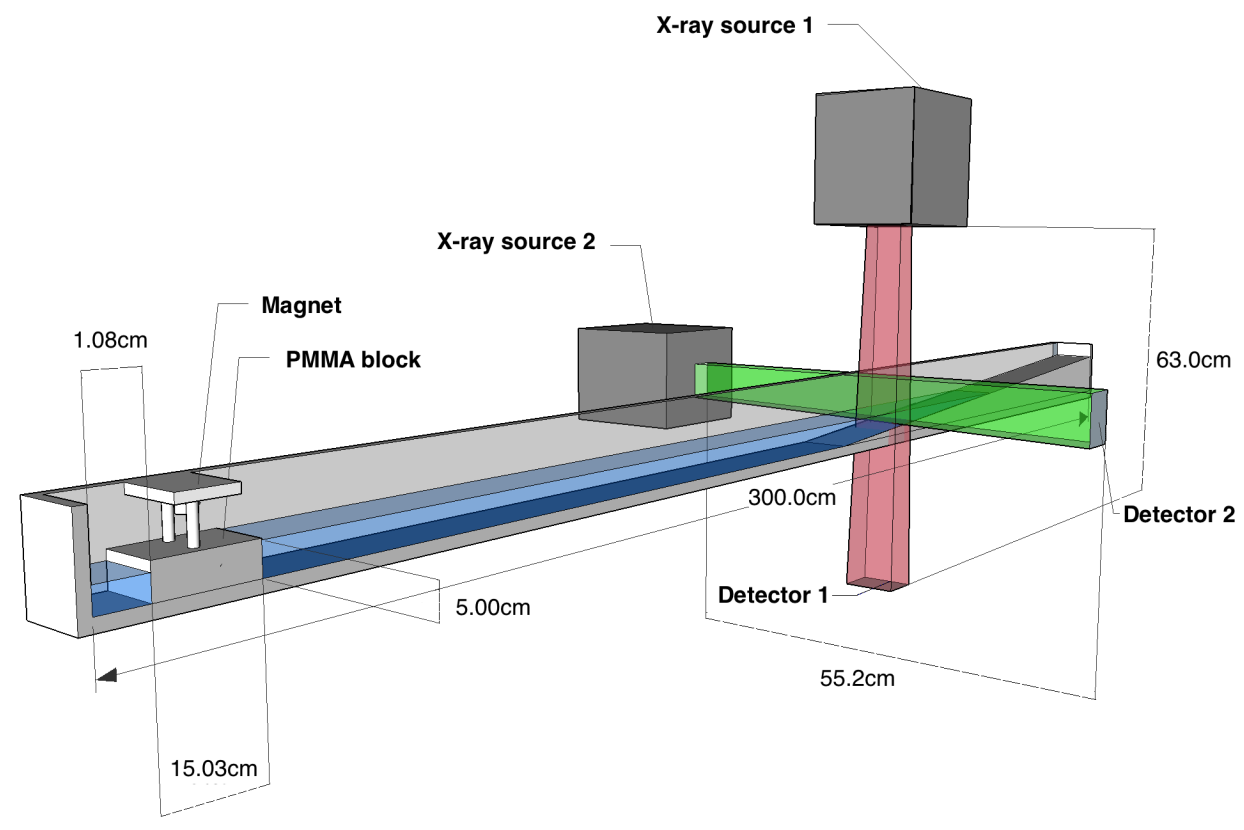

Figure 2: Positions of the wave tank and the X-ray system.

measures the incident wave height at a distance of $99.7 \mathrm{~cm}$ from the start of the wave tank. A $132 \mathrm{~cm}$ long PMMA beach, with an inclination of $5.1^{\circ}(1: 10)$ is installed in the end of the wave tank. As the solitary wave travels towards the shore, the wave steepens due to the change in water depth caused by the beach. The steep wave front develops into a plunging breaker as observed in Figure 1. To investigate the surf and the swash zone, three different field of views (FOVs) are applied by moving the position of the wave tank relative to the X-ray system, see Figure 3. A supplemental FOV is also applied further up the beach, to investigate the maximum runup. For each of the FOV including the maximum runup, three repetitions of the solitary wave were completed.

\subsection{X-ray settings and calibration}

Two perpendicular X-ray systems, similar to the X-ray tomographic X-ray system used by Hu et al. (2014) are used in this investigation. X-ray images are captured simultaneously for a horizontal and a vertical view. The voltage between the cathode and the anode in the X-ray systems is set to $80 \mathrm{kV}$ and the current is set to $5.5 \mathrm{~mA}$ for both sources. This corresponds to the maximum energy intensity available for the X-ray systems. X-rays are then generated and spread out through a collimator, resulting in a cone shaped beam. Two high-speed X-ray CMOS detectors are located on the opposite side of the X-ray sources. The detectors sensitive area is $114.9 \mathrm{~mm} \times 64.6 \mathrm{~mm}$ distributed by $1536 \times 864$ pixels. The detectors are rectangular and the $\mathrm{X}$-rays detected are therefore aligned in a rectangular based pyramid (see Figure 2). An object situated close 


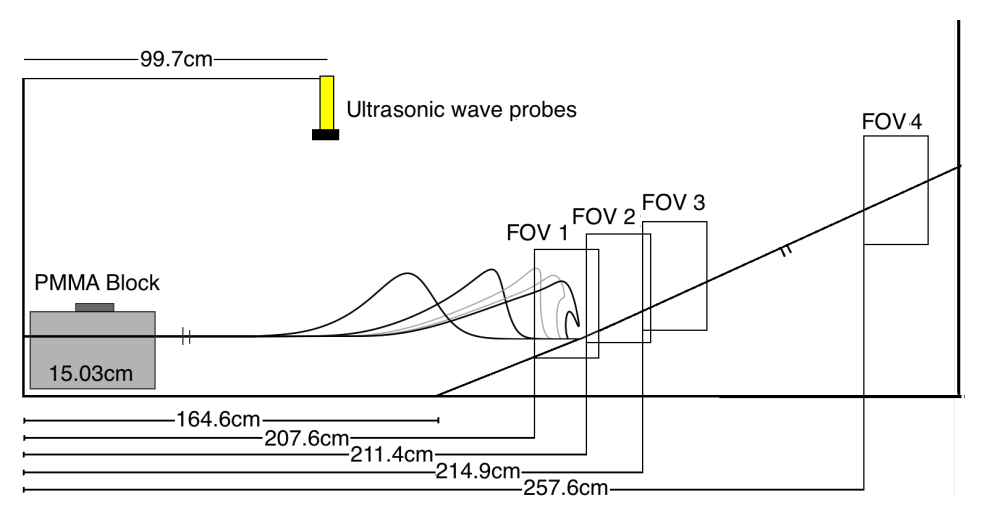

Figure 3: Experimental setup, with distance measurements.

to the X-ray source, will therefore look larger on the X-ray images, than an object that located closer to the detector. So, the size of an imaged object depends on how far it is from the detector source. To represent the correct wave height in the X-ray images, the axis on the images are scaled such that an object in the middle of the wave tank is represented in the images with its actual size. The same applies for the top view, where objects on the still water surface will be imaged with the correct size. A multi-channel trigger source synchronizes the two detectors, and images are captured at 155 frames per second (fps) with an exposure time of $6.45 \mathrm{~ms}$ with a $2 \times 2$ pixel binning, resulting in a $768 \times 432$ pixel resolution. The high speed option for the X-rays detectors is only available if the binning is performed. The X-ray intensity in an X-ray image is dependent on the amount of matter that the X-rays must penetrate through from the X-ray source to the $\mathrm{X}$-ray detector. In our experiment, the amount of X-rays attenuated is related to the amount of water that the X-rays must propagate through.

Calibration is needed for both the top and side view to estimate the amount of air and water present within the regions of the FOVs. A 3D printed box with dimensions $12 \mathrm{~cm} \mathrm{x} 10 \mathrm{~cm} \times 10 \mathrm{~cm}$ (See Figure 4) is used for the calibration of the side view. The calibration box has 4 chambers all aligned side by side with widths $6 \mathrm{~mm}, 12 \mathrm{~mm}, 24 \mathrm{~mm}$ and $48 \mathrm{~mm}$. This enables 16 different combination of water depths for the side view X-rays. In addition, a completely filled water tank gives one more calibration point. Images are captured for the 17 different water depths, and the X-ray intensity detected for each pixel is related to the water depth in the calibration box. An image of an empty wave tank is subtracted from each of the images before calibration. A piecewise linear curve is fitted to the data from the side view.

X-ray images are captured when the wave tank is filled with different levels of water to calibrate the data from the top view. The depth is varied from a still water level of $5 \mathrm{~cm}$ to a still water level of $7.5 \mathrm{~cm}$, with an increment of approximately $2.5 \mathrm{~mm}$. This gives 16 calibration points for each of the pixels in the top view detector. A second order polynomial calibration curve was fitted to data for each pixel. This was convenient, since the water path length varied from only $(0-2.5) \mathrm{cm}$, which is 5 times less than the side view variation of $(0-10) \mathrm{cm}$. So the calibration data from the top view was more rectilinear that the calibration for the side view. The second order polynomial fitting decreased the computational time significantly but introduced a small error. The average error was found to be $0.011 \mathrm{~mm}$, which is approximately 100 times smaller than the significant error related to the length measurements of this experiment. Another error associated with the X-ray measurements is the uncertainties related to the X-ray system ability to measure constant X-ray intensities for constant water depths. The error may be related to the X-ray source ability to supply a constant X-ray intensity. The maximum deviation from a mean X-ray intensity image series (300 images) was calculated to be $1.05 \%$ and $1.64 \%$ for the top and the side view, respectively. The deviations was calculated when the water depth was kept constant at $5.7 \mathrm{~cm}$.

\subsection{Air pocket detection}

Different techniques are used to detect the air pocket entrapped by the plunger for the different Xray views. The pocket captured from the side view are detected by a region growing technique where 8-connectivity is used. An example of a detected pocket is shown in the right panel of Figure 5. The region growing technique starts with a selected pixel (a seed), and checks every neighboring pixel to see if it can be 


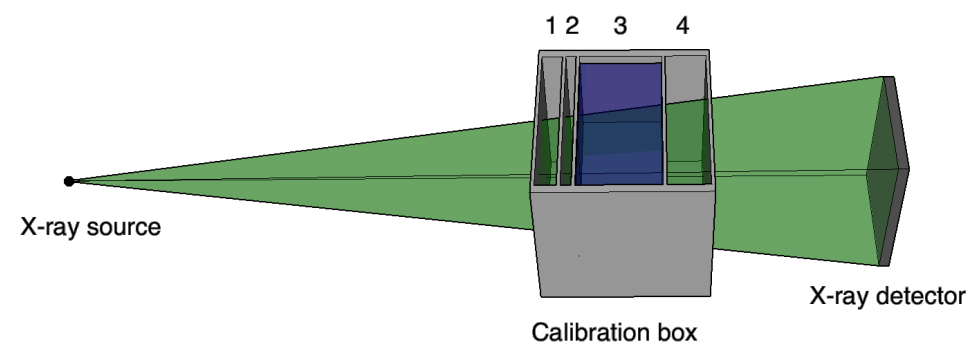

Figure 4: Calibration setup for the side view and chamber number 3 is filled with water.
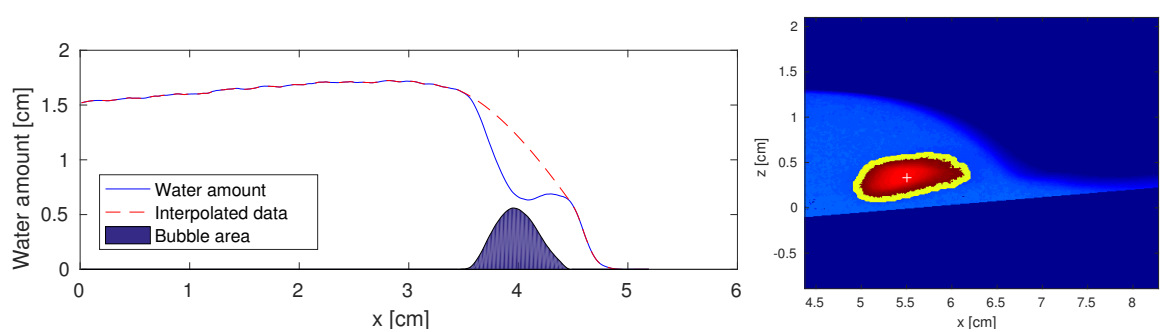

Figure 5: Examples of bubble detection. Left: The average amount of water obtained by the top view. The figure shows the void associated with the large air pocket encapsulated by the plunger. Right: Side view image of a segmented pocket. The red corresponds to the area found by the 8 connectivity region growing technique, the white cross corresponds to the seed, and the red and yellow area corresponds to the detected pocket shape and size.

labelled as an air pocket pixel or not. The criteria for a pixel to be labelled as an air pocket pixel or a wave pixel is a threshold on the pixels X-ray intensity. A pixel with an intensity that corresponds to a void of $2 \mathrm{~cm}$ is labelled as an air pocket pixel. In addition, the air pockets are not allowed to grow into areas close to the free surface and spatial limits are set to prevent this. The limits were carefully selected and should only prevent the air pockets to extend into the wave interface area. To measure the entire air entrained volume and to smooth the boundaries of the air pockets, the pockets are dilated by a disk with a diameter of 7 pixels after the detection. The area added by the dilation, is shown as yellow part in the right figure in Figure 5 .

The volume of air entrapped by the plunger can also be measured from the top view. The left panel in Figure 5 shows an example on how the volume of air is calculated. The X-ray intensity for the top view images corresponds to the height of the surface elevation. An average of the pixels over the width of the wave tank is calculated, and a profile of the average water area is obtained. The profile corresponds to the surface profile when there is no air entrained in the system, but the large air pocket marks the profile as a dimple or a cavity. To find the area occupied by the air pocket, the dimple is substituted by a line that is interpolated with a spline method. The derivative of the interpolated line is restricted to only positive values. The integrated difference between the interpolated and the actual measured profile gives the average area of air entrained in the wave. The average is then multiplied with the width of the wave tank to find the air volume. An iterative method determined the start and end points of the interpolated line. The points were chosen such that the air volume was maximized.

\subsection{D reconstruction}

A 3D reconstruction of the images captured from the side and the top view is calculated based on the reconstruction technique introduced by $\mathrm{Hu}$ et al. (2005). The volume spanned by the two X-ray systems is simplified into a cube, neglecting the cone shape of the X-rays. The cube is divided into small cells and a system of equations is solved for each detector line in the direction along the wave tank. An illustration of one line, is represented in Figure 6. One equation is needed for each of the pixels $\phi_{j}$ in the vertical detector

$$
A_{1, j} \chi_{1, j}+A_{2, j} \chi_{2, j}+\ldots \ldots .+A_{m, j} \chi_{m, j}=\phi_{j} \quad \text { for } \quad j=1 \ldots n,
$$




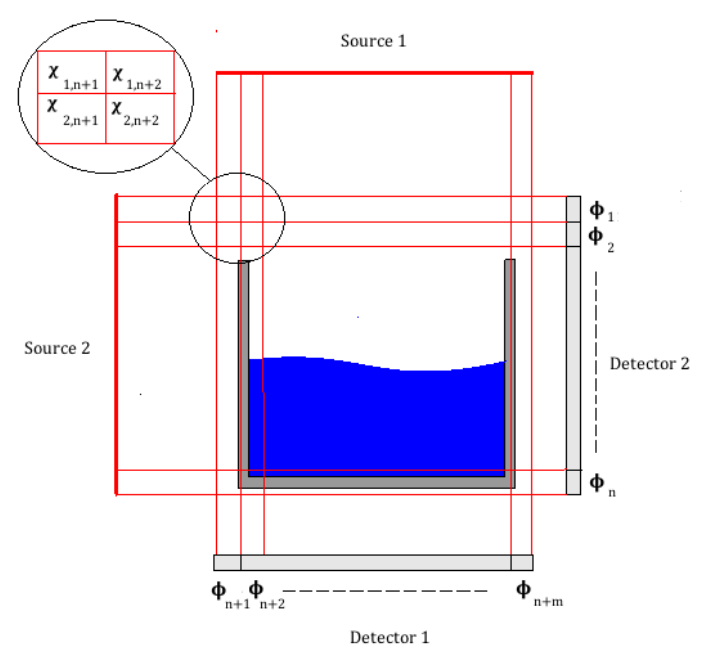

Figure 6: Sketch that shows the 3D reconstruction parameters. This describes the construction for only a single line. The reconstruction is performed for all the lines in the detectors (432).

where $A_{i, j}$ is a matrix of 1 and 0 constructed to align the sets of equations. Another set of equation is also needed for the pixels $\phi_{i+n}$ in the horizontal detector

$$
A_{i, 1} \chi_{i, 1}+A_{i, 2} \chi_{i, 2}+\ldots \ldots+A_{i, n} \chi_{i, n}=\phi_{i+n} \quad \text { for } \quad i=1 \ldots m .
$$

where $\chi_{i, j}$ is the unknown water fraction in cell $i, j$. It is a dimensionless number between 0 and 1 , where 0 is air and 1 is water. The equations forms a set of $m+n$ equation, with $n \times m$ unknowns, which is an under-determined system, and a Moore-Penrose pseudoinverse is computed to estimate the water amount $\chi_{i, j}$ from the equation set $A \chi=\phi$.

\section{Results}

All 3 repetitions for the 4 different FOVs resulted in overturning plunging breakers that impact on the dry beach. The amplitude was measured by the ultrasonic probes to be $a=2.35 \mathrm{~cm}$ at positions before the beach. The plunging breaker height $H_{p}$ was measured from the side view X-ray images to be $1.69 \mathrm{~cm}$. This gives a wave height reduction of $28 \%$.

\subsection{Incident wave}

The probe measurements are shown in Figure 7. The wave generation is repeatable for almost all the cases but the last wave in FOV 3 is bigger than the other runs. It is approximately $0.05 \mathrm{~s}$ ahead of the other waves, and approximately $10 \%$ larger. This is probably due to imperfection in the wave generation. The measurements from the last run in FOV 3 will therefore be omitted in the rest of the analysis. For all waves, the tails of the solitary waves are affected by reflections from the beach. The incoming amplitudes are probably slightly smaller than the amplitudes measured at the probe position. This has been elaborated in Smith et al. (2017) and perfect incoming solitary waves were reported in Smith et al. (2016), where no beach was mounted at the end of the wave tank.

\subsection{Result from the surf and the swash zone}

The X-ray systems are able to detect large air cavities underneath the plunging jet, and follow how they develop during runup. The X-ray images are much more detailed than the one you can retrieve with an optical camera (Smith et al., 2017). The high velocity related to the breaking process and start of the runup

of the waves, prevent the X-ray system from detecting a clear interface for smaller bubbles on milimetric scale due to blurring effects. Though, the spatial blurring does not affect the average amount of void calculated 

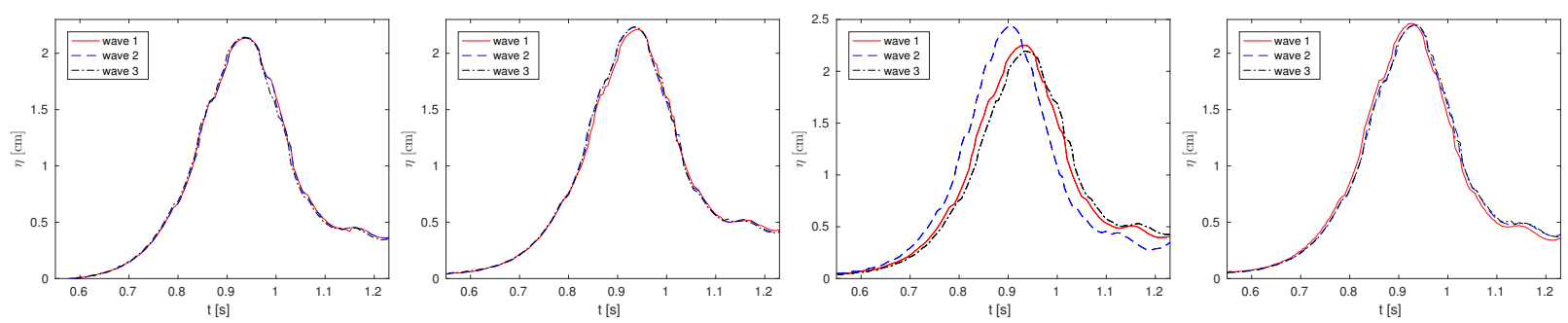

Figure 7: Ultrasonic probe measurement at position $99.7 \mathrm{~cm}$ from the start of the wave tank. For each of the different fields of view, 3 repetitions have been performed. Figures left to right corresponds to ultrasonic wave measurement for waves related to the X-ray images from FOV1, FOV2, FOV3 and FOV4 (max runup).

from the X-ray images. X-ray images from the first FOV are shown in Figure 8 and capture the waves in the surf zone. The images show an overturning jet that develops into a wave with a large air pocket encapsulated inside. $t_{0}$ is defined as the moment when the plunger hits the dry beach. In the first image from the side view $t_{-5}$, parts of the interface inside the air cavity has a vertical front and does not resemble the fine cubic curve described by Longuet-Higgins (1982). This may indicate that small parts of the wave collapse or break before the plunger hits the beach. Experiments conducted with smaller waves (not shown) show the same collapse of water inside the aerated area, but instead of a vertical front, a large blob is observed on the left hand edge of the air cavity. The collapse or instability retreats as the waves propagate on shore, and cannot be found in the next image $t_{-4}$ (not shown). The collapse or bulb may be due to surface tension and would be more prominent for smaller waves. It should also be mentioned that discrepancies between the shape of the plunger in this study and the one from Longuet-Higgins (1982) also are due differences in the water depth at the impingement. In this study the plunging jet impacts a dry beach, but in the latter, the plunging jet impacts a wave trough. A discussion of scale effects will be provided later in section 4 . The images from the top view show how a large air column is developed. The column is wider at locations close to the walls of the wave tank, and thinner in middle of the tank.

X-ray measurements from the second FOV, and the start of the swash zone are shown in Figure 9. The air cavity can clearly be seen from the side view, and it is still entrapped by water. The shape of the air tube develops from a circular shape to an elongated elliptic shape. The top view shows the same air tube shape as shown for FOV 1. The air tube diameter varies across the shore from approximately $0.5 \mathrm{~cm}$ to $1 \mathrm{~cm}$, but as time passes, the two air volumes close to the walls disintegrate into smaller air pockets and the amount of air present in the wave is asymmetric about the centerline of the wave tank.

Figure 10 shows images from FOV 3, which is located in the upper part of the swash zone. It is clear that the air disintegrates into the water, since the edges between the pocket and the water is more diffuse compared to edges at the earlier stages. However, one should keep in mind that the velocities of the air pockets are larger at this stage, which lead to a larger displacement within the exposure time, and blurring of the interfaces. Velocities as high as $70 \mathrm{~cm} / \mathrm{s}$ are measured (see Table 1), and the distance travelled in the exposure time of $6.45 \mathrm{~ms}$ is approximately $0.5 \mathrm{~cm}$. Additionally, large variations in sharpness of the boundary between the air pocket and the water was observed for the two different runs investigated at this FOV. This indicates that at this stage, the experiment is no longer repeatable.

The calculated amount of air entrapped by the plunger is visualized in the left panel in Figure 11, and listed in Table 1 and Table 2. There is no qualitative measurement from the last FOV due to difficulties in determination of the edges of the aerated area for one of the runs. The aerated volume encapsulated by the plunger is approximately $2 \mathrm{~cm}^{3}$. The center of void velocities as a function of time is shown in the right panel in Figure 11. It is clear that both the horizontal and vertical velocity increases at times after the plunge impingement. A maximum magnitude velocity of $77.6 \mathrm{~cm} / \mathrm{s}$ is obtained.

Both the images in Figure 8 and the data given in Table 1 reveal that the air pockets shape changes from an elliptic tube tilted slightly to the left, to a circular shape, and at the end, as a wide ellipse tilted to the right. The ratio between the major and minor axis of the ellipse (vortex ratio) is increasing while the segmented pocket area remains unchanged. The vortex ratio observed when the plunger impacts the beach is measured to be 1.50 and is in agreement with the linear relation found by Mead and Black (2001), $\frac{l}{w}=\frac{0.065}{m}+0.821$, where $\mathrm{m}$ is the slope of the reef or the beach. On a $5.1^{\circ}$ beach the vortex ratio is estimated 

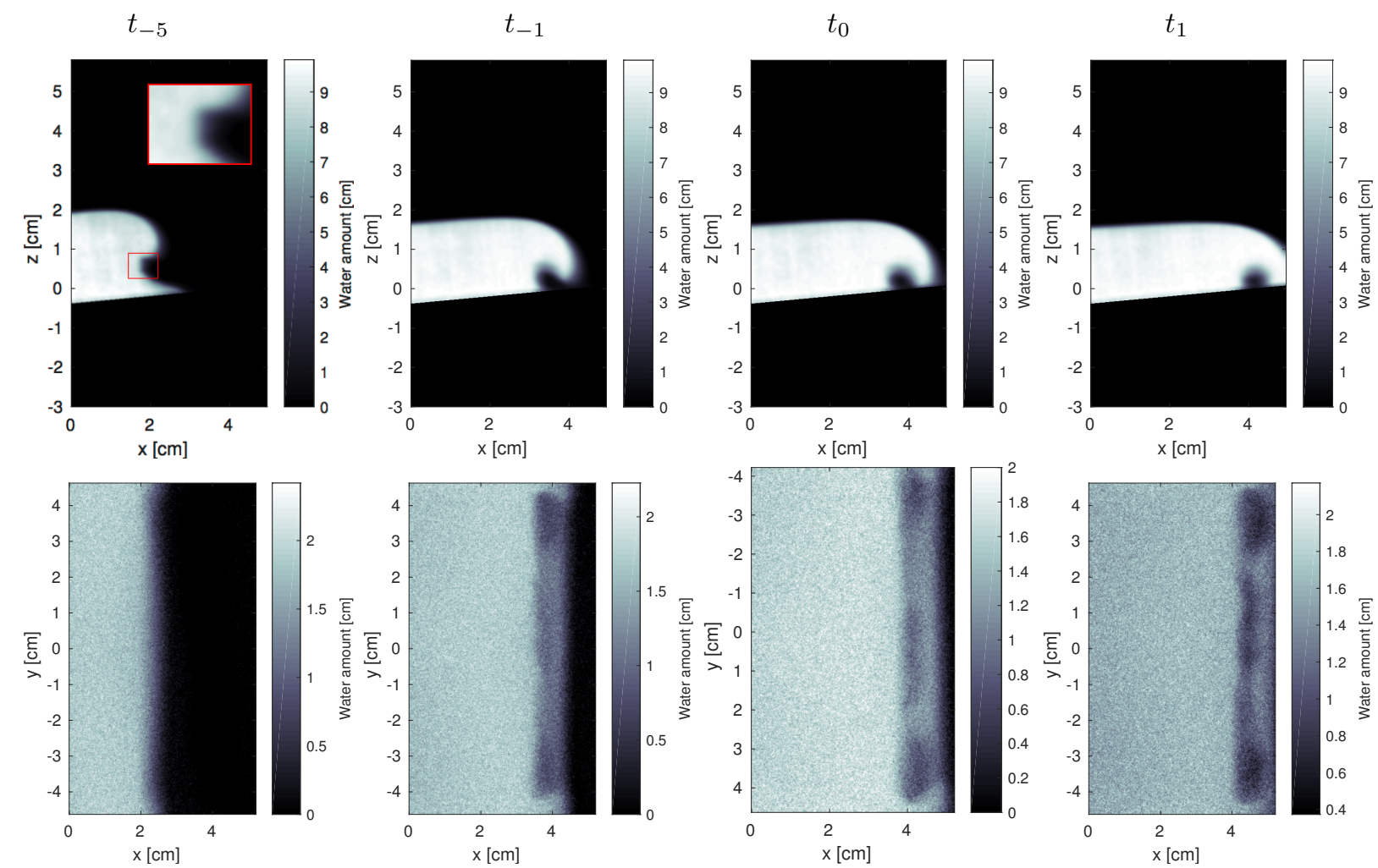

Figure 8: X-ray measurements from FOV 1, Upper images: Side view. Lower Images: Top view. $t_{0}$ corresponds to the time where the plunging jet hits the dry beach. Black corresponds to air and white corresponds to water. $\Delta t=1 / 155 \mathrm{~s}$.

$t_{2}$

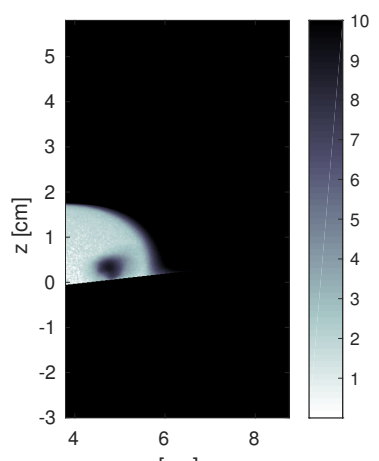

$x[\mathrm{~cm}]$

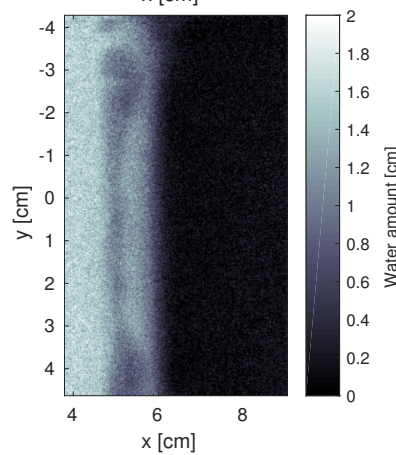

$t_{4}$

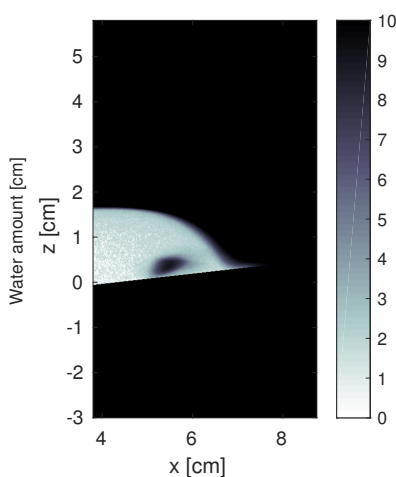

$x[\mathrm{~cm}]$

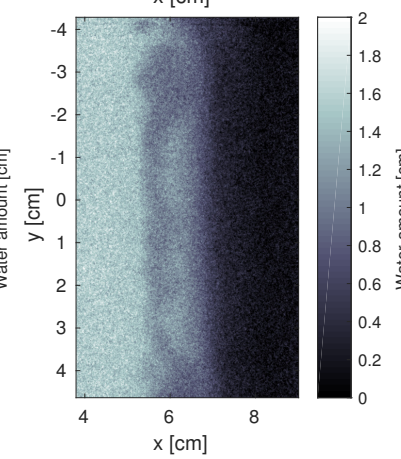

$t_{6}$
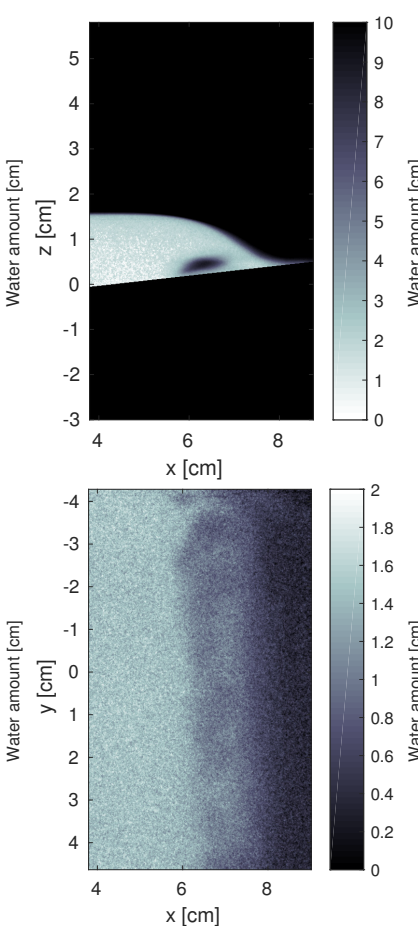

$t_{8}$
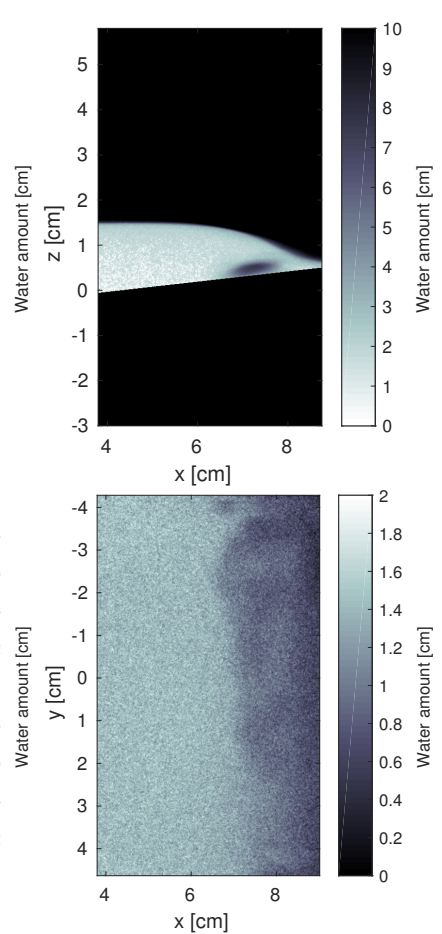

Figure 9: X-ray measurements from FOV 2, Upper images: Side view. Lower Images: Top view. $t_{0}$ corresponds to the time where the plunging jet hits the dry beach. Black corresponds to air and white corresponds to water. $\Delta t=1 / 155 \mathrm{~s}$. 

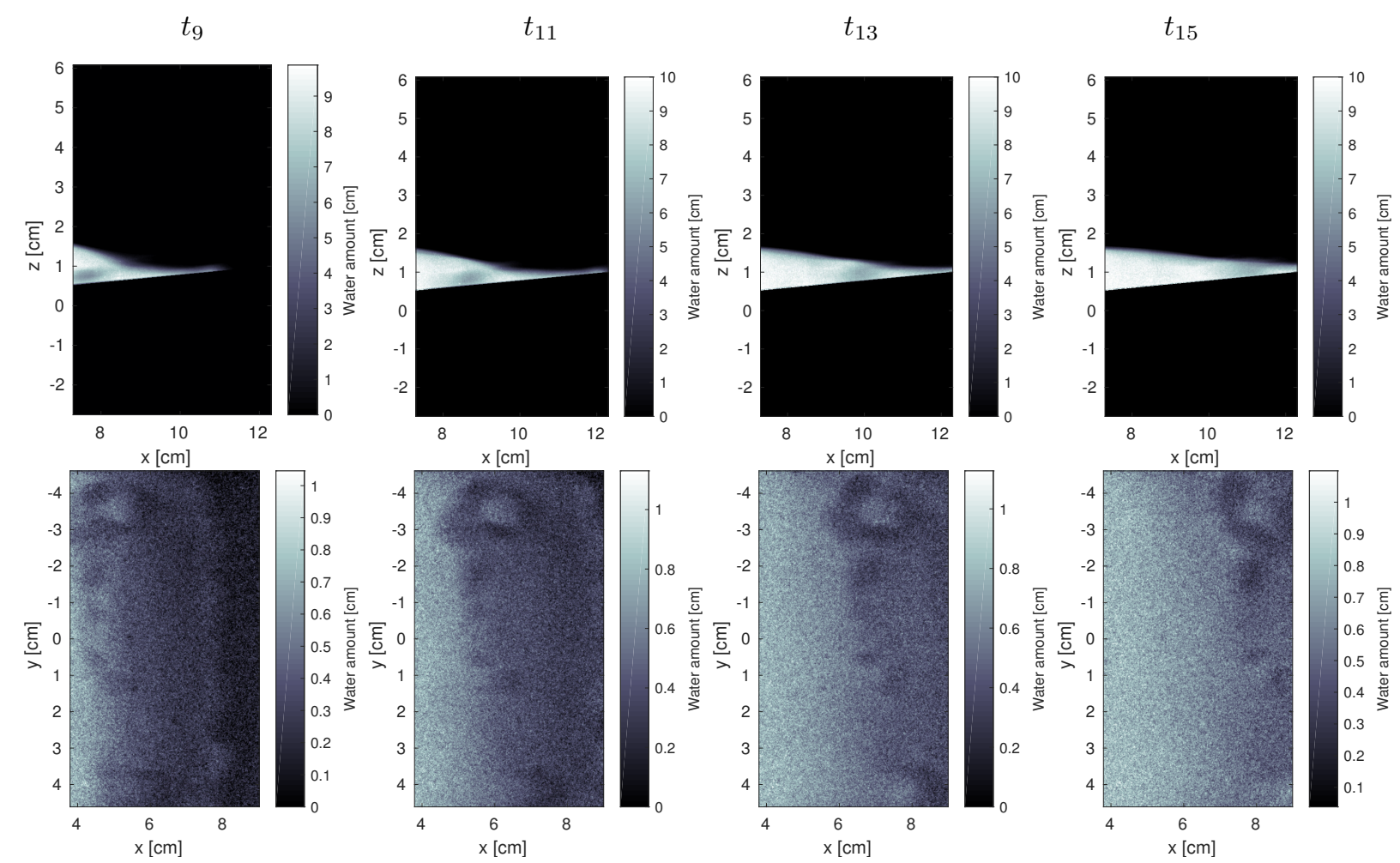

Figure 10: X-ray measurements from FOV 3, Upper images: Side view. Lower Images: Top view. $t_{0}$ corresponds to the time where the plunging jet hits the dry beach. Black corresponds to air and white corresponds to water. $\Delta t=1 / 155 \mathrm{~s}$.

\begin{tabular}{|c|c|c|c|c|c|c|c|c|c|}
\hline Time & $\begin{array}{c}\text { Volume } \\
V_{S}\left[\mathrm{~cm}^{3}\right]\end{array}$ & $\begin{array}{c}\text { Relative STD } \\
\sigma[\%]\end{array}$ & $\begin{array}{c}\text { Major axis } \\
\text { length } l[\mathrm{~cm}]\end{array}$ & $\begin{array}{c}\text { Minor axis } \\
\text { length } w[\mathrm{~cm}]\end{array}$ & $l / w$ & $\begin{array}{c}\text { Orientation } \\
\theta\left[{ }^{\circ}\right]\end{array}$ & $\begin{array}{c}\text { Area } \\
A\left[\mathrm{~cm}^{2}\right]\end{array}$ & $\begin{array}{c}\text { Velocity } \\
V_{x}[\mathrm{~cm} / \mathrm{s}]\end{array}$ & $\begin{array}{c}\text { Velocity } \\
V_{z}[\mathrm{cmps}]\end{array}$ \\
\hline$t_{0}=0.00 \mathrm{~ms}$ & 2.48 & 1.3 & 1.14 & 0.76 & 1.50 & -0.40 & 0.65 & & \\
\hline$t_{1}=6.45 \mathrm{~ms}$ & 2.29 & 1.5 & 1.00 & 0.81 & 1.23 & 9.3 & 0.64 & 51.5 & 0.61 \\
\hline$t_{2}=12.90 \mathrm{~ms}$ & 2.30 & 0.7 & 1.11 & 0.70 & 1.59 & 16.4 & 0.62 & 51.1 & 0.0 \\
\hline$t_{3}=19.35 \mathrm{~ms}$ & 2.26 & 1.8 & 1.21 & 0.64 & 1.89 & 13.9 & 0.61 & 52.8 & 1.4 \\
\hline$t_{4}=25.80 \mathrm{~ms}$ & 2.26 & 2.4 & 1.28 & 0.61 & 2.10 & 13.1 & 0.62 & 54.9 & 2.9 \\
\hline$t_{5}^{t}=32.25 \mathrm{~ms}$ & 2.26 & 3.2 & 1.34 & 0.60 & 2.23 & 12.2 & 0.64 & 60.3 & 4.0 \\
\hline$t_{6}=38.70 \mathrm{~ms}$ & 2.22 & 4.1 & 1.40 & 0.58 & 2.41 & 11.4 & 0.64 & 65.4 & 4.4 \\
\hline$t_{7}=45.15 \mathrm{~ms}$ & 2.12 & 5.6 & 1.44 & 0.56 & 2.57 & 10.3 & 0.64 & 71.5 & 4.8 \\
\hline$t_{8}=51.60 \mathrm{~ms}$ & 2.04 & 5.1 & 1.51 & 0.56 & 2.70 & 10.4 & 0.67 & 77.4 & 5.6 \\
\hline
\end{tabular}

Table 1: Air pocket characteristics obtained from the side view

to be 1.47. Blenkinsopp and Chaplin (2008) found a relation between the major axis length, the minor axis length, and area spanned by the air cavity, $A=\frac{2 \sqrt{3}}{5} w l$. Our measurement agrees with the formula, and deviates only by $8 \%$.

The length of the aerated area measured from the top view is smaller than the major axis length obtained from the side view. This is expected since the cavities are slightly tilted in respect to the top view. The standard deviation $\sigma$ is larger for the top view than for the side view, and is probably due to inaccuracy in the determination of the amount of water absent due to the present of air in the water. It should be mentioned that the volume error $\sigma$ in Tables 1 and 2 are larger than the error associated with the other quantities, since the total error increases with the number of dimensions in the measurements.

The development of the surface elevations and the air cavities for all the FOVs are shown in Figure 12. The shape of the two last cavities, $t_{10}$ and $t_{11}$, are collected from the first run in the last FOV. The surface elevation contour plot at times before the plunger impinges the beach resembles the shapes presented in Smith et al. (2017). Nevertheless, the interface is determined by the maximum gradient magnitude of a black and white image of the data, and 3D effects and droplets may be neglected. The void center marked on the air cavities in Figure 12 are located higher than the spatial center for the later plots, and indicate that the air is biased towards the surface inside the aerated area. 

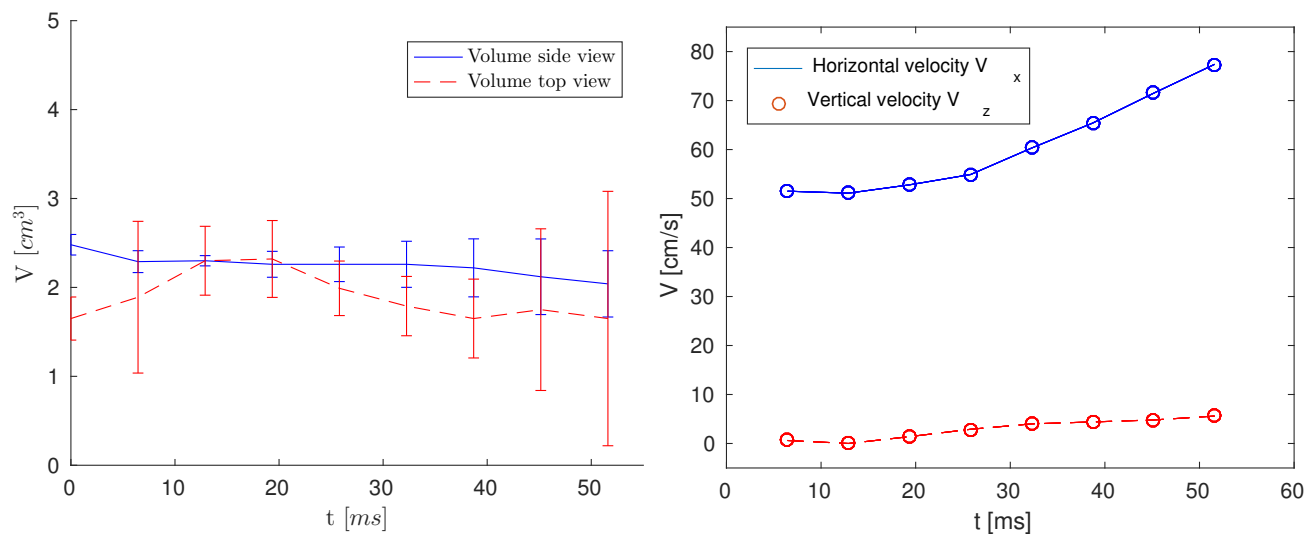

Figure 11: Left: Aerated volume entrapped by the wave as a function of time. The error bars are the 0.975 confidence interval of the mean. Right: The void center velocity measured as a function of time, from the side view.

$\begin{array}{cccc}\text { Time } & \text { Volume } V_{t}\left[\mathbf{c m}^{3}\right] & \text { Relative STD } \sigma[\%] & \text { Length } l_{t}[\mathbf{c m}] \\ t_{0}=0.00 \mathrm{~ms} & 1.65 & 4.1 & 0.75 \\ t_{1}=6.45 \mathrm{~ms} & 1.89 & 12.6 & 1.06 \\ t_{2}=12.90 \mathrm{~ms} & 2.30 & 4.7 & 1.24 \\ t_{3}=19.35 \mathrm{~ms} & 2.32 & 5.2 & 1.39 \\ t_{4}=25.80 \mathrm{~ms} & 1.99 & 4.3 & 1.49 \\ t_{5}=32.25 \mathrm{~ms} & 1.79 & 5.3 & 1.61 \\ t_{6}=38.70 \mathrm{~ms} & 1.65 & 7.5 & 1.64 \\ t_{7}=45.15 \mathrm{~ms} & 1.75 & 14.5 & 1.81 \\ t_{8}=51.60 \mathrm{~ms} & 1.65 & 24.2 & \end{array}$

Table 2: Air pocket characteristics obtained from the top view
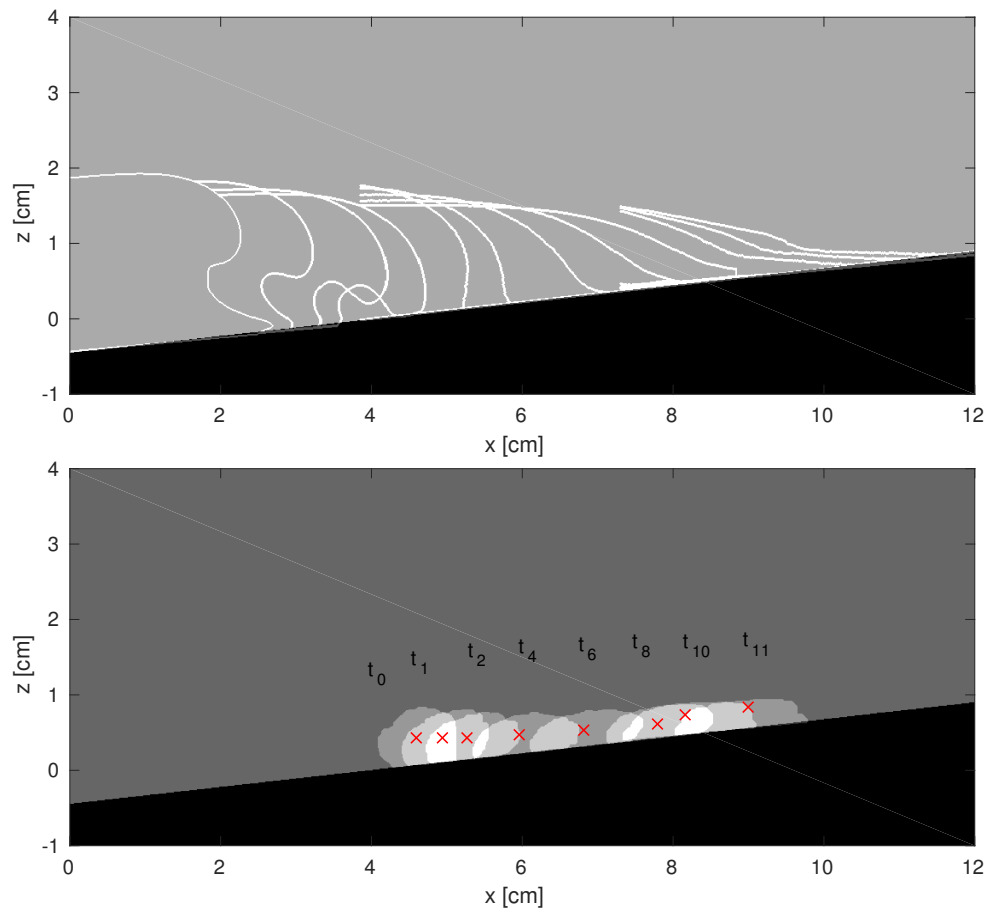

Figure 12: Upper Figure: Assembled surface profiles from the three different fields of view. Lower Figure: Assembled air pocket measurements from the three different FOVs. The red crosses give the locations of the void center of the air pockets. 

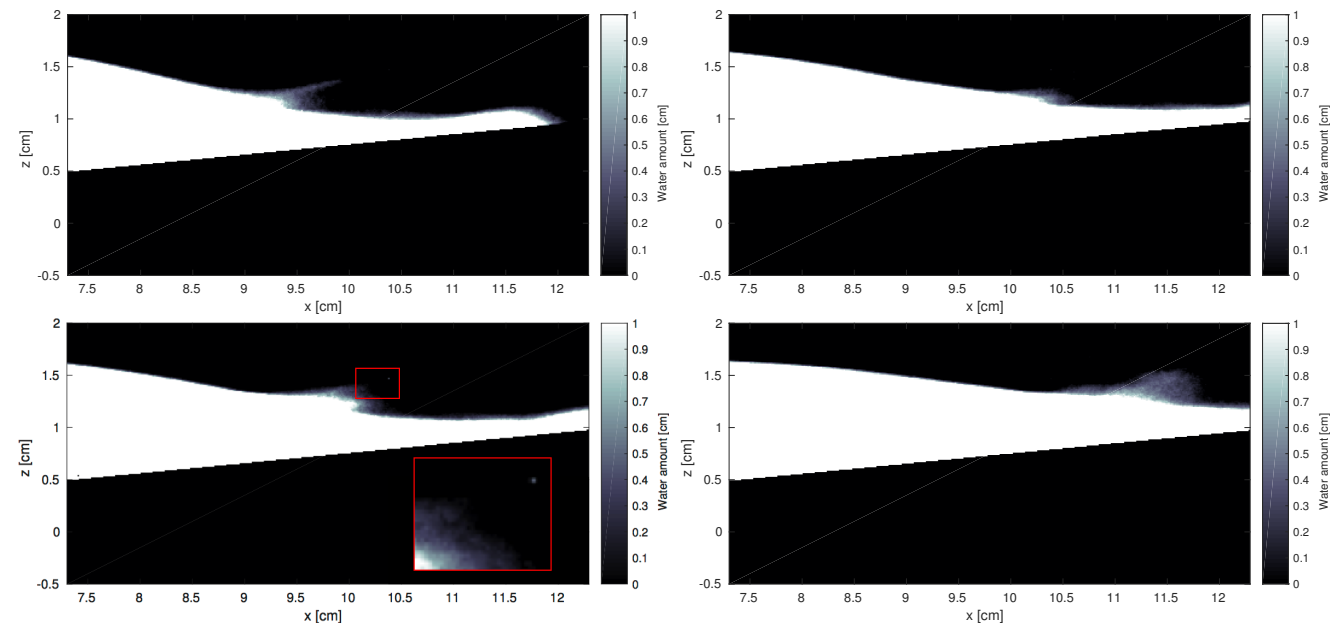

Figure 13: Images from FOV 3 where the contrast is enhanced with respect to water in the air phase. Upper Figure: Images from the first run at $\mathrm{t}=t_{10}$ and $\mathrm{t}=t_{12}$. Lower Figure: Images from the last run, $t=t_{11}$ and $t=t_{14}$.

Images from the last FOV where the contrast is shifted such that water in the air phase is enhanced are shown in Figure 13. The images reveal that not only is the air entrained in the water, but a small amount of water is also mixed in the air phase. This can originate from several mechanisms such as secondary breaking, re-splashing, sprays, or bubble bursting. The shape of the interface in the left images can be associated with the liquid film and droplet generated and reported by Veron et al. (2012). In the lower left image a tiny water droplet is erupted from the disturbed area at the interface. The difference between the runs in Figure 13 implies that the air entrainment and swash tongue evolution are not repeatable at this stage.

\subsection{Maximum runup}

Cross shore images (top view) and side view images of the maximum runup are shown in Figure 14. The maximum runup for the three different runs was $(53.20,53.16,51.65) \mathrm{cm}$. The shape of the runup tongues was curved for all of the runs and two of the runs have air bubbles present at the center of the half circled runup tip. The second run has two air bubbles located on top of the thin liquid film. The two bubbles merged into one bubble right after reaching maximum runup (not shown). The diameter of the two large air bubbles for the first and the second run are approximately $1.2 \mathrm{~cm}$, which correspond to a half sphere with a volume of $0.45 \mathrm{~cm}^{3}$. This is approximately $25 \%$ of the initial air volume entrapped by the plunger. The runup thickness of the liquid film without the air bubbles was approximately $5 \mathrm{~mm}$ and the width of the film was estimated to $3 \mathrm{~cm}$. The presence of large air bubbles at the incident of maximum runup implies that the inundation time is short relative to the time it takes for the bubble to burst. This is a feature that is limited to small scale wave breaking, and is not scalable to larger waves. The roundness of the shoreline is due to friction along the sidewalls of the wave tank, and is also more prominent for small scale experiments.

\section{4. $3 D$ reconstruction of the surface}

3D-reconstructed fields are shown in Figure 15 and reveal the evolution from an overturning jet into an encapsulated air pocket in 3D. There are some errors with the 3D reconstruction from the first FOV. It appears in the right corner in the first and second images in Figure 15. Water is equally distributed into the pixels in the vertical column. This is clearly an artifact that might be due to misalignment of the wave tank in relation to the X-ray system.

\section{Scale effects in breaking events}

Surface tension plays an important role in small scale wave breaking events (Stagonas et al., 2011), in particularly for the formation of the plunger, the bubbles lifetime, and the amount of air entrained by the waves. It is clear from the thickness and roundness of the plunger that surface tension is significant in this 

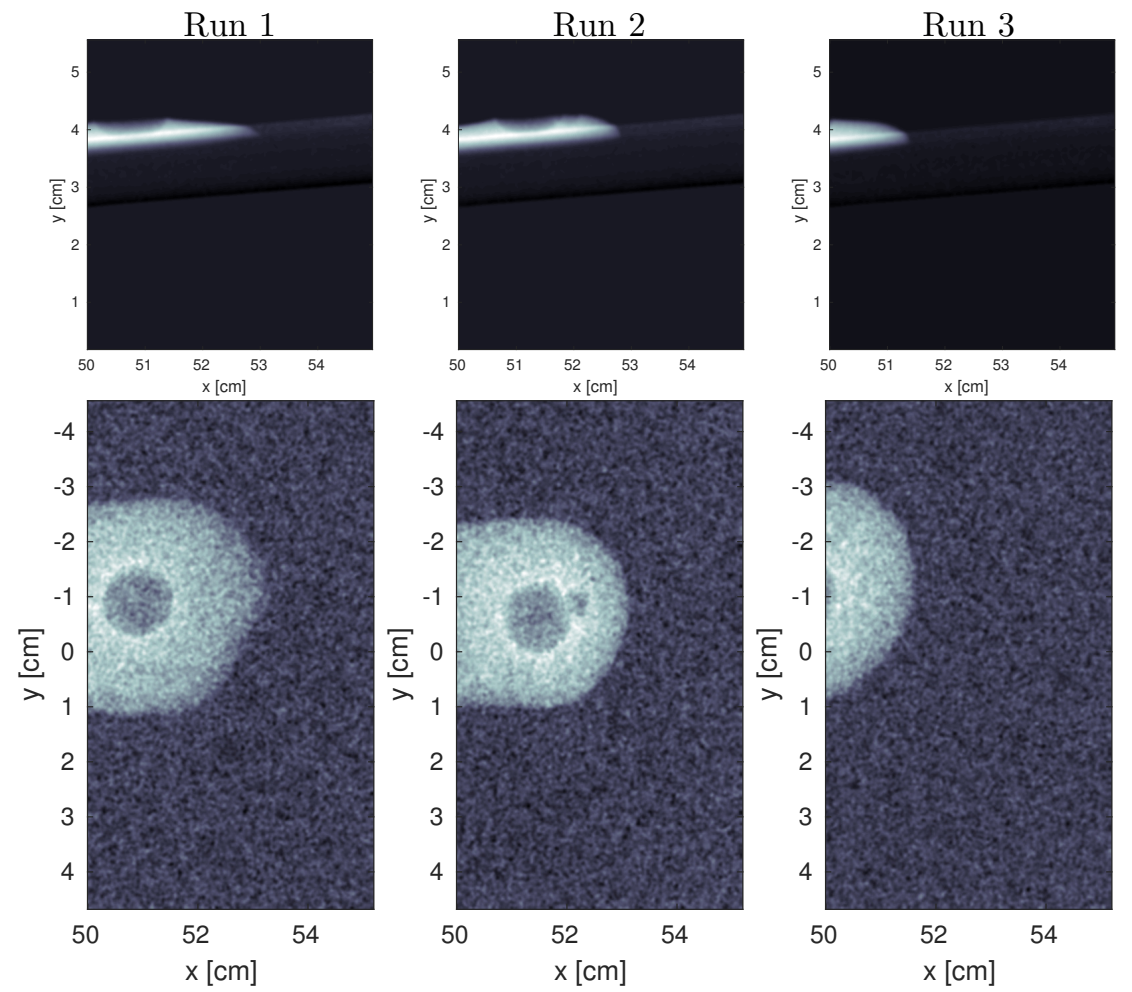

Figure 14: Images of the maximum runup for three different runs. The upper figures show the side view and the lower figures show the top view. The white in the image corresponds to water and the black corresponds to void.
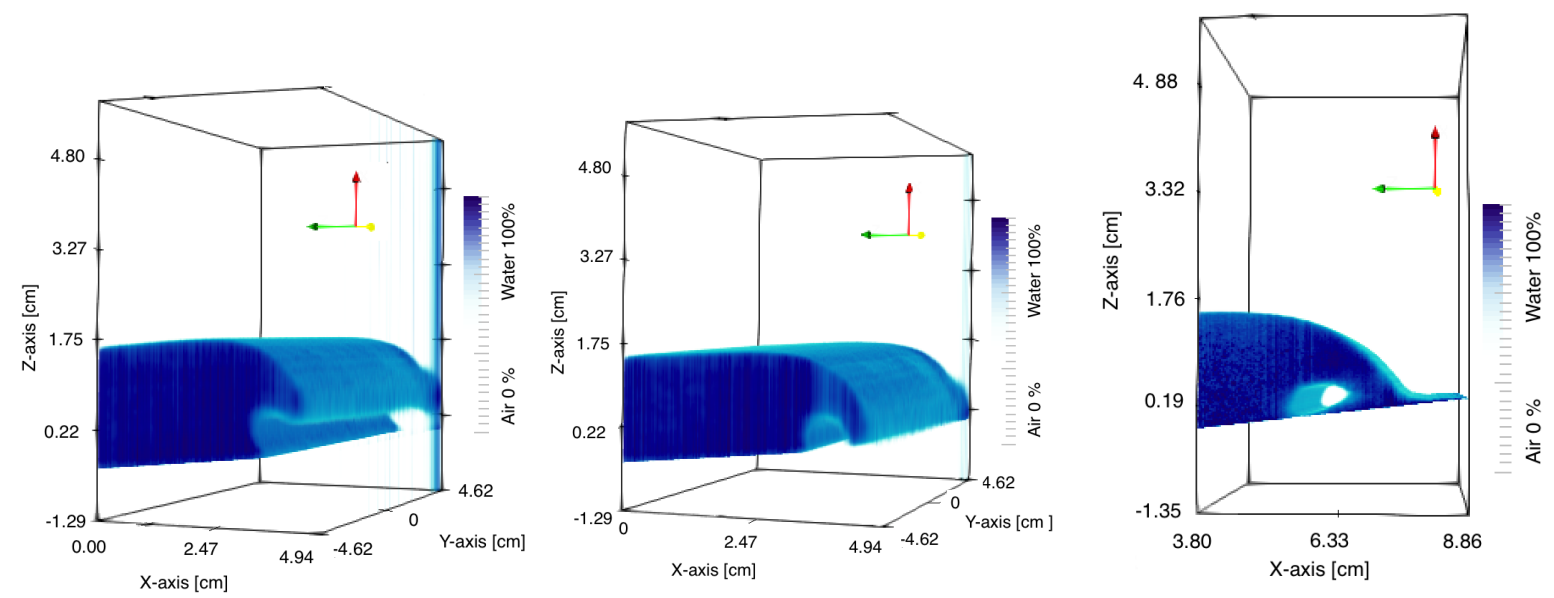

Figure 15: Images from the 3D-reconstruction. Left: FOV $1, t=t_{-2}$. Middle: FOV $1, t=t_{0}$, Right: FOV2, $t=t_{4}$. The blue data corresponds to water and white data corresponds to air. 
study. Studies by Couriel et al. (1998) counclude that the large air cavities formed by breaking waves are not affected by surface tension if the breaker height $H_{p}$ is larger than $25 \mathrm{~mm}$. In this study the breaker height was measured to be $16.9 \mathrm{~mm}$, and surface tension effects are therefore important in the experiments conducted. The Bond number $B o=\frac{\Delta \rho g H_{p}^{2}}{\sigma}$ was calculated to be 38.4. The effect of increasing the surface tension forces relative to the gravitational force (reducing the Bond number $\mathrm{Bo}$ ) is numerically studied by Song and Sirviente (2004). They found that an increase in the strength of the surface tension decreased the breaking jet intensity and decreased the amount of air encapsulated by the plunger. The lowest Bond number they investigated was $B o=38$ with a wave slope of $\xi=0.55$ and a Reynolds number $R e=350$. The wave did not develop into a plunging breaker at all, and did not resemble the overturning jet observed in this study. The Reynolds number related to the solitary waves in this study, can be approximated to $R e=\frac{u_{w} \lambda_{w}}{\nu} \approx 3 \cdot 10^{5}$, where $u_{w}=\sqrt{g(H+a / 2)}$. The approximate solitary wave length $\lambda_{w}$, is defined as $\lambda_{w}=2 u_{w} t_{w}$, where $t_{w}$ is the time it takes for the solitary waves to halve its amplitude from it's maximum. The difference between the computational and experimental data reveals that the viscous effects are of great importance in the development of plunging breakers. However, in our study the viscous effects are negligible due to the high Reynolds number. Blenkinsopp and Chaplin (2008) investigated plunging breakers with a breaker height larger than $50 \mathrm{~mm}$, and could therefore eliminate surface tension. They found that the volume of air encapsulated by the plunger is geometrical scalable. So the $8 \%$ deviation from the linear relationship found by Mead and Black (2001) are mostly due to surface tension. The scale effects of the distribution of air bubble after wave breaking are studied in Blenkinsopp and Chaplin (2011). The results show that the distribution of air bubbles remains the same regardless of scale. The study also discusses the effect of using freshwater instead of seawater to investigate ocean wave breaking. They found similarities in the void fraction obtained for the cases but the amount of smaller air bubbles was larger for the seawater case. The time it takes for the bubbles to rise to the surface $t_{b}$ is clearly not dependent on breaking, but on the size of bubbles and the distance from the surface. A comparison of the runup for this study and the larger scaled runup from Smith et al. (2017) reveals that $t_{b}$ is not scalable with the maximum runup time, since all the air had escaped long before maximum runup occurred. Even though, one might expect that the small swash flow depth in this study would decreases the time it takes for all the bubbles to escape the surface, but the development of a thin liquid film, where surface tension dominates, makes the bubbles attach to the surface for a long period.

\section{Conclusion}

Succsessful small scale experiments of breaking solitary waves were visualized by a novel X-ray technique. The use of an ultra fast X-ray system made it possible to investigate plunging breakers in great detail. The breaking characteristics measured in this study comply with the measurements from Blenkinsopp and Chaplin (2008). The solitary waves generated developed into plunging breakers, and encapsulated a large air tube of approximately $2 \mathrm{~cm}^{3}$. Velocity measurements of the void center revealed that the air tube accelerated upward the beach, and velocities as high as $77.6 \mathrm{~cm} / \mathrm{s}$ were reported. The shape of the air tube, obtained from the side view, developed from an elongate elliptic shape tilted slightly to the left to a circular shape, and then to an elongated elliptic shape tilted to the right. The top view images showed two larger air cavities with a thin air tube connecting them, located close to the walls of the wave tank. Further up the beach, dissimilarities between the runs were reported. The experiments revealed that air is entrained in the water phase, but also that the water is present in the air phase. There are some scale effects present in this study, but the experiment could not be performed at a larger scale, due to limitations of the X-ray system strength and design. However, the design of the experiment can be scaled up and larger breaking waves, e.g. the waves generated in Smith et al. (2017) (waves with an amplitude of approximately $10 \mathrm{~cm}$ ) can be used and provided physical measurements that are applicable for breaking waves in the ocean. This is the first study where an X-ray system is utilized to investigate solitary breaking waves. The novel, high speed, high resolution X-ray tomography system demonstrated here shows great promise as a tool for investigating other types of bubbly flows, and for providing future reference data for numerical and mathematical modellers. 


\section{Acknowledgement}

This work was funded by the Research Council of Norway through the research project DOMT - Developments in Optical Measurement Technologies (project number 231491).

\section{References}

Banner, M., Peregrine, D., 1993. Wave breaking in deep water. Annual Review of Fluid Mechanics 25 (1), 373-397.

Blenkinsopp, C., Chaplin, J., 2007. Void fraction measurements in breaking waves. In: Proceedings of the Royal Society of London A: Mathematical, Physical and Engineering Sciences. Vol. 463. The Royal Society, pp. 3151-3170.

Blenkinsopp, C., Chaplin, J., 2008. The effect of relative crest submergence on wave breaking over submerged slopes. Coastal Engineering 55 (12), 967-974.

Blenkinsopp, C., Chaplin, J., 2011. Void fraction measurements and scale effects in breaking waves in freshwater and seawater. Coastal Engineering 58 (5), 417-428.

Chang, K.-A., Liu, P. L.-F., 1998. Velocity, acceleration and vorticity under a breaking wave. Physics of Fluids 10 (1), 327-329.

Couriel, E., Horton, P., Cox, D., 1998. Supplementary 2-d physical modelling of breaking wave characteristics. Technicial Report, Water Research Laboratory, TR98-14.

Grilli, S., Svendsen, I., Subramanya, R., 1997. Breaking criterion and characteristics for solitary waves on slopes. Journal of waterway, port, coastal, and ocean engineering 123 (3), 102-112.

Heindel, T. J., Gray, J. N., Jensen, T. C., 2008. An x-ray system for visualizing fluid flows. Flow Measurement and Instrumentation 19 (2), 67-78.

Hu, B., Langsholt, M., Liu, L., Andersson, P., Lawrence, C., 2014. Flow structure and phase distribution in stratified and slug flows measured by x-ray tomography. International Journal of Multiphase Flow 67, $162-179$.

Hu, B., Stewart, C., Hale, C. P., Lawrence, C. J., Hall, A. R., Zwiens, H., Hewitt, G. F., 2005. Development of an x-ray computed tomography (ct) system with sparse sources: application to three-phase pipe flow visualization. Experiments in fluids 39 (4), 667-678.

Iafrati, A., 2009. Numerical study of the effects of the breaking intensity on wave breaking flows. Journal of Fluid Mechanics 622, 371-411.

Jensen, A., Pedersen, G. K., Wood, D. J., 2003. An experimental study of wave run-up at a steep beach. Journal of Fluid Mechanics 486, 161-188.

Kiger, K. T., Duncan, J. H., 2012. Air-entrainment mechanisms in plunging jets and breaking waves. Annual Review of Fluid Mechanics 44, 563-596.

Lamarre, E., Melville, W., 1991. Air entrainment and dissipation in breaking waves. Nature 351 (6326), 469.

Lin, C., Kao, M.-J., Tzeng, G.-W., Wong, W.-Y., Yang, J., Raikar, R. V., Wu, T.-R., Liu, P. L.-F., 2015. Study on flow fields of boundary-layer separation and hydraulic jump during rundown motion of shoaling solitary wave. Journal of Earthquake and Tsunami 9 (05), 1540002.

Longuet-Higgins, M. S., 1982. Parametric solutions for breaking waves. Journal of Fluid Mechanics 121, 403-424.

Mead, S., Black, K., 2001. Predicting the breaking intensity of surfing waves. Journal of Coastal Research, $51-65$. 
Pedersen, G., Lindstrøm, E., Bertelsen, A., Jensen, A., Laskovski, D., Sælevik, G., 2013. Runup and boundary layers on sloping beaches. Physics of Fluids (1994-present) 25 (1), 012102.

Peregrine, D. H., 1983. Breaking waves on beaches. Annual Review of Fluid Mechanics 15 (1), 149-178.

Siddiqui, M. K., Loewen, M. R., Richardson, C., Asher, W. E., Jessup, A. T., 2001. Simultaneous particle image velocimetry and infrared imagery of microscale breaking waves. Physics of Fluids 13 (7), 1891-1903.

Smith, L., Hu, B., Kolaas, J., Jensen, A., Sveen, J. K., 2016. X-ray ptv measurement of solitary waves. In: 18th International Symposium on the Application of Laser and Imaging Techniques to Fluid Mechanics. ISBN 978-989-98777-8-8, pp. $2604-2612$.

Smith, L., Jensen, A., Pedersen, G., 2017. Investigation of breaking and non-breaking solitary waves and measurements of swash zone dynamics on a $5^{\circ}$ beach. Coastal Engineering 120, 38-46.

Song, C., Sirviente, A. I., 2004. A numerical study of breaking waves. Physics of fluids 16 (7), 2649-2667.

Stagonas, D., Warbrick, D., Muller, G., Magagna, D., 2011. Surface tension effects on energy dissipation by small scale, experimental breaking waves. Coastal Engineering 58 (9), 826-836.

Veron, F., Hopkins, C., Harrison, E., Mueller, J., 2012. Sea spray spume droplet production in high wind speeds. Geophysical Research Letters 39 (16).

Wellington, S., Vinegar, H., et al., 1987. X-ray computerized tomography. Journal of Petroleum Technology 39 (08), 885-898. 